\title{
The Antikythera Mechanism: A Computer Science Perspective
}

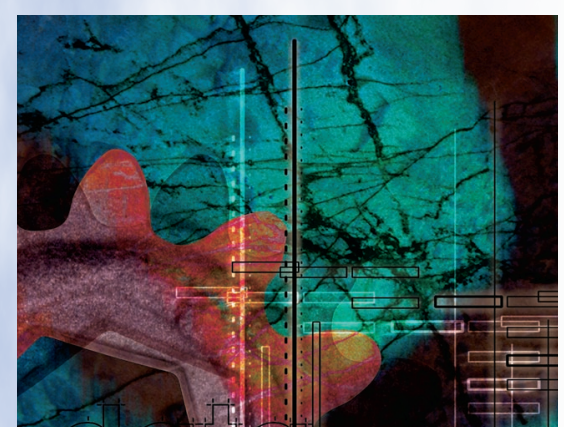

Diomidis Spinellis

Athens University of Economics and Business

The Antikythera mechanism

is an ancient astronomical

calculator that contains a

lunisolar calendar, predicts

eclipses, and indicates

the moon's position and

phase. Its use of multiple

dials and interlocking gears

eerily foreshadows modern

computing concepts from

the fields of digital design,

programming, and software

engineering.

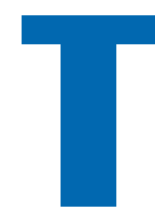

wo thousand years separate us from an ancient Greek computing device known as the Antikythera mechanism. Here I explain the mechanism's operation based on its reconstruction in Squeak Etoys, a multimedia authoring environment primarily designed to help high school students learn scientific and engineering concepts. ${ }^{1,2}$ The reconstruction relies on the recent findings that an international cross-disciplinary team of scientists obtained through surface imaging and high-resolution x-ray tomography. My work aims to present the functioning of this remarkable device using working code, the language of our community.

The complete image of this implementation is available online as open source software running on the Etoys environment (www.dmst. aueb.gr/dds/sw/ameso). I encourage readers to download an Etoys image and run the software on it, as they step through the descriptions in this article.

\section{HISTORY}

In 1900, a group of sponge fishers seeking shelter from the Kythera Sea's cruel weather anchored their boats on the barren island of Antikythera. Continuing their diving there, they discovered at a depth of 42 meters an ancient shipwreck with bronze and marble statues. For almost a year afterward, they worked with the Greek government to salvage the ship's contents. These artifacts were then transferred for preservation and study to the National Archaeological Museum in Athens, where they remain on display to this day. Among the recovered items, which dated from the first century B.C., were a beautiful nude bronze statue and a severely corroded lump of bronze clearly containing gear wheels.

Numerous scientists have devoted their lives to the study of this mysterious mechanism. Based on the few legible letters in fragments and descriptions of mechanical contraptions in ancient Greek and Roman texts, it was initially identified as an astrolabe or planetarium. Derek J. de Solla Price, the father of scientometrics, subsequently spent three decades analyzing and reconstructing the device. Using radiographs, he was able to count the teeth of most of the device's gears and construct a detailed model of their operation. In his seminal 1974 monograph, "Gears from the Greeks," he described the mechanism as a calendar computer. ${ }^{3}$ Famously, his proposed model included a differential mechanism, similar to the one found in the drive trains of modern cars, apparently constructed scores of centuries before its reinvention. 


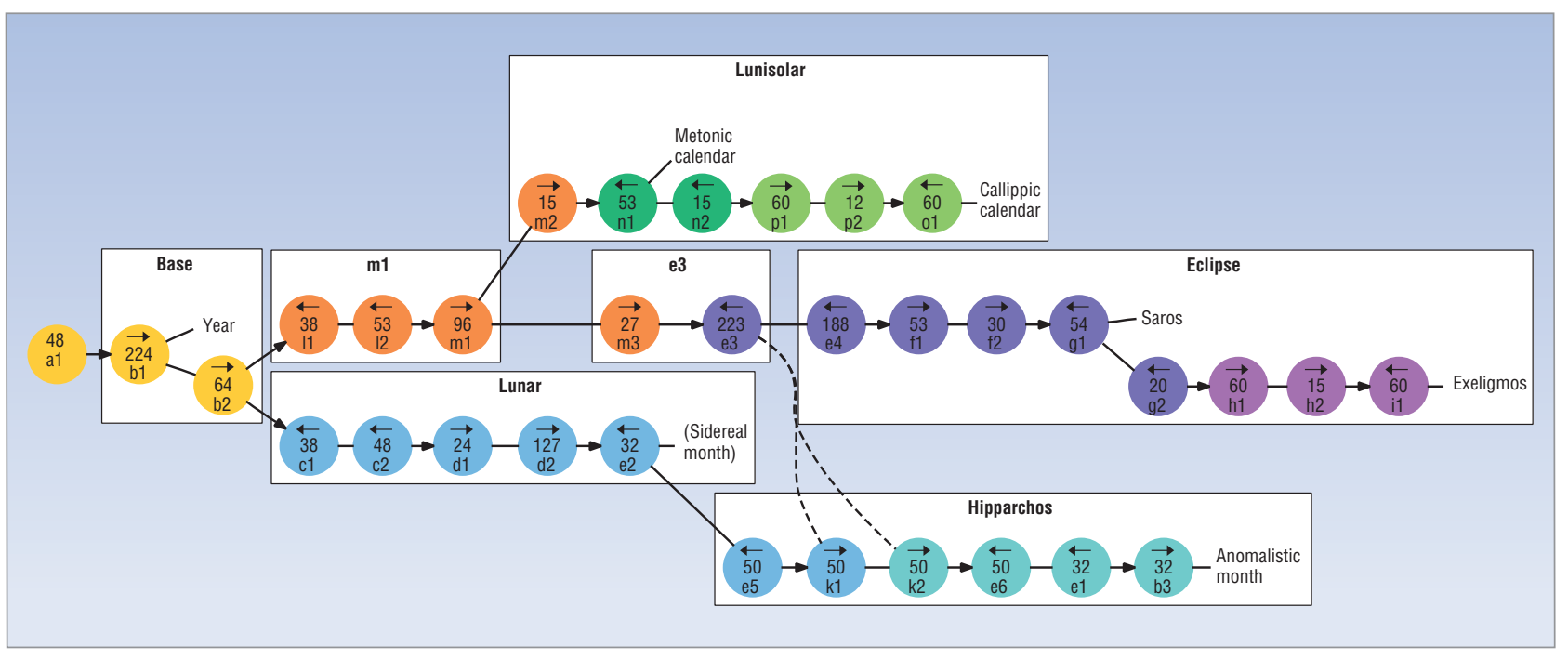

Figure 1. Antikythera mechanism operational model. Each circle represents a gear, with the arrow at the top indicating the direction the gear is turning, the number in the center how many teeth the gear has, and the letter-number combination at the bottom the name of the gear. Gears with the same letter are concentric, and numbers increase from the front to the back of the mechanism.

Recently, astronomers, archeologists, computer engineers, and physicists from around the world collaborated on the Antikythera Mechanism Research Project (www. antikythera-mechanism.gr) to reconstruct a more precise model. They used three computer-based imaging techniques-3D x-ray microfocus computed tomography, polynomial texture mapping, and digitized highquality photography-to study virtual cross-sections of the device under various simulated lighting conditions (samples of the images are available for interactive study at the project website). The project's results, published in Nature in November 2006, ${ }^{4}$ confirmed that the device was indeed a calendar computer. However, the new model proposes that the gears Price identified as a differential instead operate in a distinct, but no less sophisticated, manner to calculate the anomaly in the moon's rotation.

\section{CALCULATING WITH GEARS}

The Antikythera mechanism is believed to consist of 35 gears. Archeologists identified 30 in the surviving fragments, while science historian Michael Wright and the authors of the Nature study introduced another five to explain the device's functionality. ${ }^{4,5}$

Figure 1 shows the relationship of the gears, each represented by a circle. The arrow at the top of each circle indicates the direction the gear is turning: $\rightarrow$ for clockwise and $\leftarrow$ for counterclockwise. The number in the center indicates how many teeth the gear has. All studies of the mechanism name gears systematically with a letter-number combination; the figure adopts the nomenclature used in the Nature article: Gears with the same letter are concentric, and numbers increase from the front to the back of the mechanism. ${ }^{4} \mathrm{~A}$ simple line connects gears that rotate together as one piece, while an arrow connects those that engage other gears through their teeth. By making a pair of gears with your hands and using your fingers as teeth, you can easily verify that when two gears engage, their direction of rotation reverses.

When two gears engage through their teeth, such as b2 with c1, their ratio of rotation periods will equal the ratio of their teeth. Thus, b2 will complete one rotation in 64 time units, whereas c1 will complete one rotation in 38 time units. Concentric gears rotating together have by definition the same rotation period. Therefore, obtaining the ratio derived by a sequence of rotating gears requires multiplying together the ratios of all engaged gears.

Input to the Antikythera mechanism is controlled through gear a1, which engages laterally with gear b1 and could conceivably be rotated with a hand-crank. However, the actual value of the mechanism's input appears through gear b1, which, via a dial on the mechanism's front face, shows the sun's position throughout the year on the Zodiac cycle and a 365-day calendar (Figure 2a). The calendar's scale can be rotated by one day every four years to take into account leap years. The known computed outputs of the mechanism are

- a lunisolar calendar (based on lunar months),

- a prediction of eclipses, and

- the moon's position and phase.

The lunisolar calendar and the eclipse prediction dials appear on the device's back face (Figure 2b), while the moon's position and phase appear on the front face. All dials rotate clockwise, and this is why on Figure 1 the gears driving the back-face dials rotate counterclockwise. 


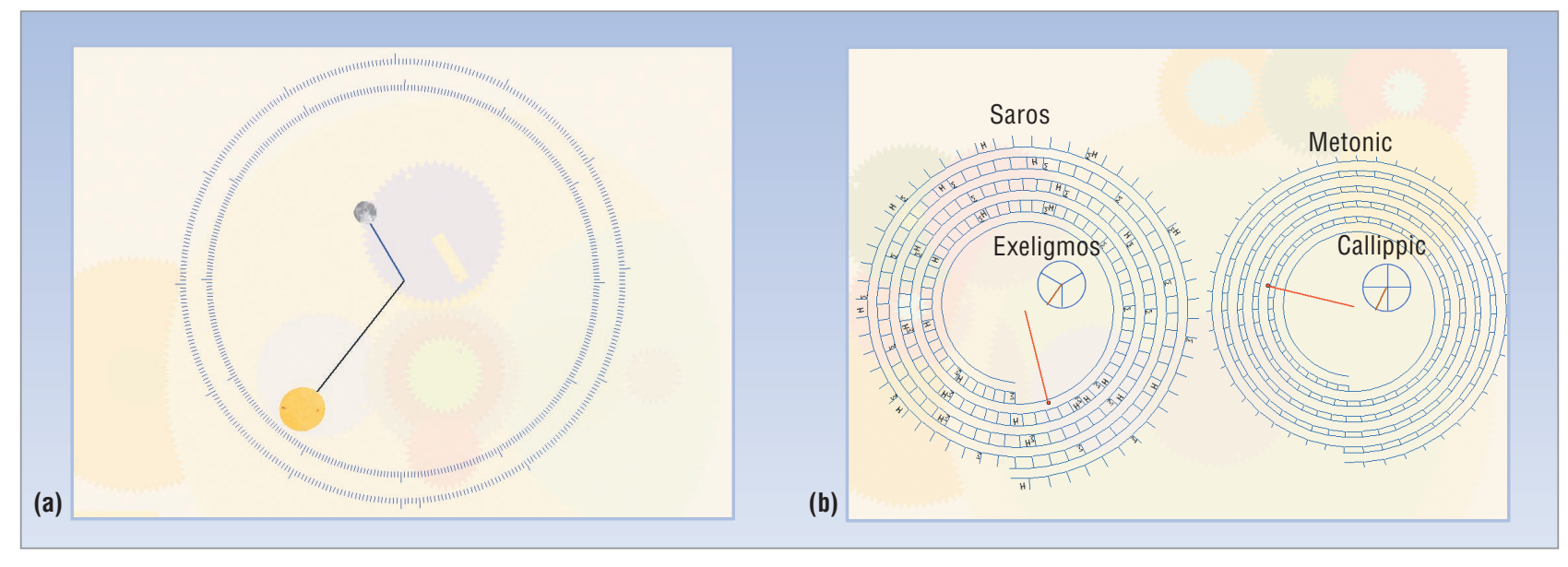

Figure 2. Antikythera mechanism's front and back faces. (a) A dial on the front face shows the sun's position throughout the year on the Zodiac cycle and a 365-day calendar. (b) The back face contains two lunicalendar dials (showing the Metonic and Callippic cycles) and two eclipse-prediction dials (showing the Saros and Exeligmos cycles).

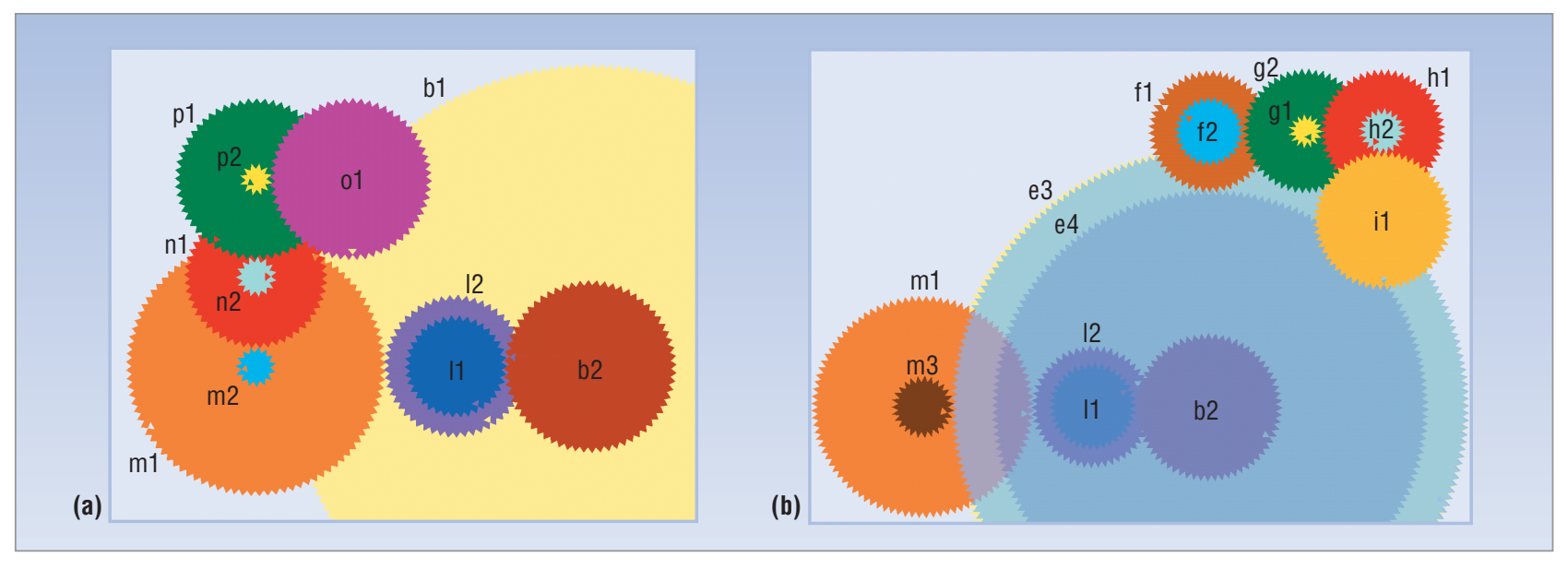

Figure 3. Emulator setup of (a) lunisolar calendar and (b) eclipse-prediction gears.

\section{LUNISOLAR CALENDAR}

Reckoning time progression through the moon's phases is convenient. It involves a calendar based on the visibly recurring lunar phases defining the 291/2-day synodic month-the time from one full moon to the next. However, the months of such a calendar don't fit correctly in the seasonal year, which has practical significance for, say, agriculture.

In the fifth century B.C., the Athenian astronomer Meton devised a way around this problem by observing that 19 seasonal (known as tropical) years contain almost exactly 235 synodic months, and proposing a cycle containing 125 full months of 30 days and 110 hollow, 29day, months. ${ }^{6}$ A century later, Callippus further refined that model by proposing the removal of one day every four Metonic cycles. Two dials on the back of the Antikythera mechanism indicate each month in a Metonic cycle as well as track progress through the Callippic cycle.

To increase the Metonic display's resolution, the dial rotates five times in each cycle with a pointer tracking a five-turn spiral. As the pointer rotates, the spiral's grooves force it to move toward the outer turns of the spiral, similarly to a needle tracking a gramophone record. Once the pointer reaches the end of the spiral, the human operator would presumably return it to the beginning.

Gear n2 driving the Metonic calendar's dial must rotate 5 times in 19 years, thus the ratio between gear b1 tracking the tropical years and $\mathrm{n} 2$ should be $5 / 19$. Indeed, the sequence b2-11-12-m1-m2-n1 calculates this ratio: $64 / 38 \times 53 / 96 \times 15 / 53=960 / 3648=5 / 19$. Further, gear o 2 driving the Callippic cycle's dial must turn at $1 / 20$ of the Metonic dial: once every four cycles of five turns each. The sequence n2-p1-p2-o1 calculates the required ratio: $15 / 60 \times 12 / 60=1 / 20$. Figure 3 a shows the emulator setup of these gears.

\section{ECLIPSE PREDICTION}

The Antikythera mechanism predicts eclipses by means of the Saros cycle established by ancient Babylonian astronomers: a period of 223 and $1 / 3$ synodic 


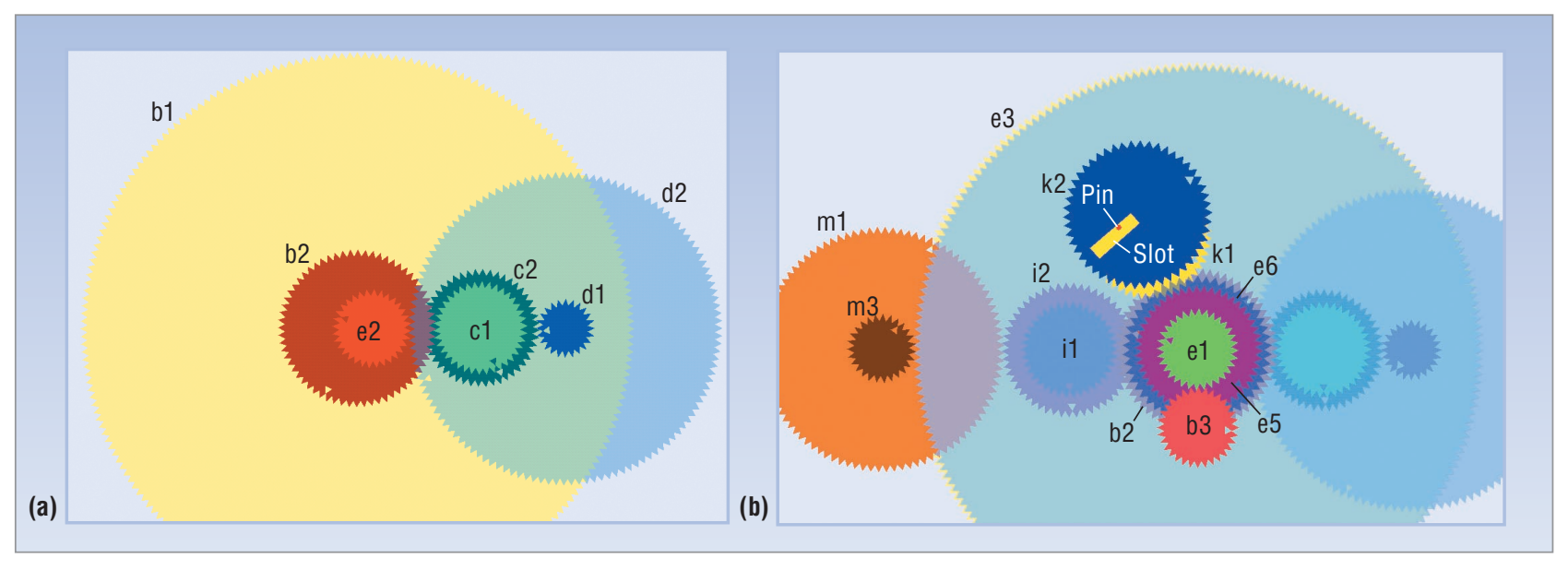

Figure 4. Emulator setup of gears to (a) calculate the sidereal month and (b) modify this calculation using Hipparchos's lunar mechanism to model the moon's elliptical orbit.

months in which identical moon and sun eclipses occur. Glyphs on the 223-month divisions of the plate where the Saros dial rotates indicate each eclipse type. The letter $\Sigma$ (for $\Sigma \mathrm{E} \Lambda \mathrm{HNH}$ - moon) indicates a lunar eclipse, while the letter $\mathrm{H}$ (for $\mathrm{H} \Lambda \mathrm{IO} \Sigma$-sun) a solar one. Like the Metonic display, the Saros display is laid out in a fourturn spiral. Interestingly, its construction involves two elements of modern software engineering: the use of a lookup table (the Saros eclipse data) to aid computation, and the adoption of a design pattern (a spiral for increasing the display's resolution).

Because the Saros cycle contains a 1/3-day fraction, it's necessary to wait three Saros cycles to witness an eclipse at the same time. Thus, a separate dial indicates the Exeligmos cycle, which comprises three Saros cycles and can be used to predict the time of each eclipse.

Figure $3 \mathrm{~b}$ shows the emulator setup of the eclipseprediction gears. We already know from the Metonic calendar that there are 235 synodic months in 19 years. For the Saros display, we need four revolutions in 223 synodic months, a ratio of $4 / 223 \times 235 / 19$. The sequence b2-11-12-m1-m3-e3-e4-f1-f2-g1 establishes this ratio, which can be easily verified with a calculator. Further, the Exeligmos dial must turn once every three four-turn Saros cycles, thus at a rate 1/12 of Saros. The sequence g2-h1-h2-i1 calculates this ratio.

\section{LUNAR CALCULATIONS}

The Antikythera mechanism's front dial indicates the moon's anomalistic month-its position on the celestial sphere taking into account both the moon's elliptical orbit and the additional rotation of the ellipse's two extreme points. This anomaly is caused by the solar tide, and one full rotation takes nine years to complete. Gears b0 and q1 combine the moon's position with that of the sun to show the moon's phase. The three-step calculation of the moon's position is the most sophisticated of the mechanism's known parts.
The first step involves calculating the sidereal month, the moon's period in a fixed frame of reference. In a period of 19 years, the moon performs 235 synodic rotations (from the Metonic calendar) and another 19 due to its rotation around the sun-a total of 254 . The sequence b2-c1-c2-d1-d2-e2, shown in Figure 4a, calculates the required yearly rotation ratio 254/19.

Next, the Antikythera mechanism models the moon's elliptical orbit through an ingenious device known as Hipparchos's lunar mechanism. The sidereal rotation established on gear e2 is transferred to gear e 5 , which is mounted on the same axle, as Figure $4 \mathrm{~b}$ shows. Gear e5 in turn turns $\mathrm{k} 1$, which has a pin mounted a small distance from its center. Gear k2 is mounted below k1, but its center is slightly displaced from k1's center. The pin moves within a slot cut into gear $\mathrm{k} 2$, and, because the two gears are eccentrically mounted, harmonically varies k2's rotation rate. Running the Antikythera emulator demonstrates that $\mathrm{k} 2$ 's rotational speed is high when the slot is at the top of the screen and low when it's at the bottom; this models the corresponding variation of the moon's speed between its perigee and apogee.

Finally, the Antikythera mechanism models the rotation of this elliptical orbit by mounting $\mathrm{k} 1$ and $\mathrm{k} 2$ on e3. The gear e 3 rotates at the rate of the elliptical orbit's rotation-the precession period of the moon's long axis-through the sequence b2-11-12-m1-m3-e3. Note that this rotates $\mathrm{k} 2$ 's axis and thereby complicates driving a dial with it. Thus, $\mathrm{k} 2$ drives $\mathrm{e} 6$, which is on a fixed axis. Gear e6 in turn drives e1, which is located on the front-dial side of e3, and b3 moves the rotation clockwise to the front dial's center. In computer engineering terms, the sequence e6-e1-b3 interfaces the processing unit to the display unit.

Another parallel with modern computing technology is the dual role of some gears: $\mathrm{e} 3$ in the calculation of both the Saros and the anomalistic month, and $\mathrm{m} 1$ in the calculation of Saros and the lunisolar calendar. This is 
a technique commonly employed in digital circuits-for example, in a full-adder implementation, the half-adder sum output typically drives both the sum and the carrybit circuits.

\section{ETOYS IMPLEMENTATION}

The Web offers numerous Antikythera mechanism simulators based on Price's outdated model that replicate the mechanism's operation in software using computer arithmetic to simulate the gear interactions, and then reflect back the computed results onto the simulated gears. In contrast, my implementation emulates the modern model; it doesn't calculate the gear positions but instead employs mechanical principles (gear teeth pushing each other) to emulate the mechanism at a physical level. This approach let me experience the mechanism's physical properties and appreciate the thinking of its ancient developer. It also fitted neatly with the Etoys platform's constructivist learning approach.

Etoys programming is based on assembling primitives that manipulate visual objects appearing on the screen. The intellectual forebears of Etoys are Logo, Smalltalk, and HyperCard. Etoys is currently used as a teaching vehicle in high schools in Asia, Europe, Japan, South America, and the United States. It's also an integral part of the One Laptop per Child (http://laptop.org/laptop) initiative's XO computer, known as the "\$100 laptop."

Each gear and dial appearing on the screen is a separate object. Although the gears might look formidable, building them was simple: I constructed them as polygons, tooth by tooth. I started with an empty polygon. After I added the tooth's two sides, I rotated the gear by the tooth's displacement and repeated the operation. This mode of construction resembles the manual cutting of physical gear teeth. Having the gears as polygons makes modeling their interactions child's play. Etoys has a built-in primitive to locate overlapping objects. Thus, on each time step, I simply look for overlapping polygons and rotate them in the appropriate direction until they no longer overlap.

I used a similar principle to implement the spiral dials' pointer-follower mechanism and the pin-and-slot device of Hipparchos's lunar mechanism. The emulated backface dials contain a small circular pin at their end that traces the spirals. When the pin overlaps with the spiral's edge, I simply increase the dial's length and move the pin to its new end. To implement Hipparchos's lunar mechanism, I bore a hole on the polygon of gear $\mathrm{k} 2$ by adding 6 more vertices. The pin is a separate object, which I rotate in sync with $\mathrm{k} 1$. Whenever the pin overlaps $\mathrm{k} 2$, $\mathrm{k} 2$ rotates, thus responding to k1's "push."

For simplicity, the emulation and corresponding images in this article depart from the device's construction in a few ways. First, the positioning of the gears and dials doesn't follow the mechanism's actual layout, because positioning gears on the horizontal and vertical axes is significantly simpler, and it also makes the gears' operation easier to inspect. Further, although in the mechanical operation as gears engage their direction reverses, in my implementation I hard-coded the appropriate new direction in each gear-engagement test.

Finally, in contrast to the actual mechanism, in my implementation the ratio of gear teeth to gear diameter is constant. Because I had already simplified the gear layout, I didn't see any point in following the exact gear dimensions. The fixed-sized teeth and the liberties I've taken with gear placement result in some gears that should be mounted on the same axis appearing in places different from the original. This isn't a problem in the emulator because I implement gears mounted on top of each other by simply maintaining them on the same heading after each gear rotation.

In the actual mechanism, varying teeth sizes allows gear rotations representing different calculated values to appear on the same axis for further processing. For example, in the original mechanism, the sequence b2i1-i2-m1-m3-e3 representing the rotation of the moon's orbit line of apses (perigee and apogee) shares the same axis with the sequence b2-c1-c2-d1-d2-e2 representing a sidereal month. This allows the two quantities to be subtracted-through the epicyclical mounting of $\mathrm{k} 1$ and k2 - to calculate the moon's eccentric motion at the anomalistic month's required rate.

Here, we can draw an analogy with hardware construction for high-speed computers. The Antikythera mechanism employs different gear sizes to drive two separate quantities to the same axis. Modern printed circuit board design software similarly routes separate but related signal paths in a way that keeps their length equal, ensuring that the signals will arrive concurrently at their destination for further processing.

$\mathbf{E}^{2}$ mulating rather than simulating the Antikythera mechanism proved to be a sound choice. For example, it revealed two bugs that would have otherwise gone unnoticed. My first operation of the back-face dials had the Saros dial scan months at a different rate than the Metonic dial. Normally both should move through lunar months at exactly the same rate: 235 months every 19 years. Further, the Callippic dial appeared to be moving too slowly. By examining the number of teeth I had programmed in each gear, I found that gear $\mathrm{p} 2$ had 15 teeth instead of 12 and gear g1 had 60 teeth instead of 54 . This accident demonstrates that faithfully emulating the mechanism makes it possible to validate its operation.

Adopting the Etoys platform made development of the emulator a productive, accessible, and enjoyable activity. Scientific and engineering visualization applications can be a tough nut to crack, and have attracted the attention of many influential researchers. ${ }^{7,8}$ Cookie-cutter techniques and frameworks such as those for implementing desktop applications are ineffective at presenting dispa- 
rate phenomena that can't be standardized to a specific format. Moreover, to be accessible to students, educational applications should avoid complex algorithms, formulas, or techniques.

Having experimented with other candidate environments like Visual Basic and Tcl/Tk, I can vouch that Etoys currently holds a significant edge in its niche. It let me appreciate the mechanism's beauty and, I dare say, even "talk" with that unknown computer engineer who two millennia ago was grappling with similar problems.

\section{Acknowledgments}

This work was partially funded by the European Community's Sixth Framework Programme under contract IST-2005-033331, "Software Quality Observatory for Open Source Software (SQO-OSS).” Many thanks to Tony Freeth and the Antikythera Mechanism Research Project team for supplying a high-resolution photo of the back dial.

\section{References}

1. D. Ingalls et al., "Back to the Future: The Story of Squeak, a Practical Smalltalk Written in Itself," ACM SIGPLAN Notices, vol. 32, no. 10, 1997, pp. 318-326.

2. M. Gaelli, O. Nierstrasz, and S. Stinckwich, "Idioms for Composing Games with EToys," Proc. 4th Int'l Conf. Creating, Connecting and Collaborating through Computing, IEEE CS Press, 2006, pp. 222-231.
3. D. de Solla Price, "Gears from the Greeks: The Antikythera Mechanism-a Calendar Computer from Ca. 80 B.C.,” Trans. Am. Philosophical Soc, new series, vol. 64, part 7, 1974.

4. T. Freeth et al., "Decoding the Ancient Greek Astronomical Calculator Known as the Antikythera Mechanism," Nature, vol. 444, no. 7119, 2006, pp. 587-591.

5. M.T. Wright, "The Antikythera Mechanism: A New Gearing Scheme,” Bull. Scientific Instrument Soc., vol. 85, 2005, pp. 2-7.

6. N. Dershowitz and E.M. Reingold, Calendrical Calculations, Cambridge Univ. Press, 1997.

7. B.W. Kernighan, "Experience with Tcl/Tk for Scientific and Engineering Visualization," Proc. 3rd Ann. Tcl/Tk Workshop, vol. 3, Usenix Assoc., 1995, pp. 269-278.

8. C. Reas and B. Fry, Processing: A Programming Handbook for Visual Designers and Artists, MIT Press, 2007.

Diomidis Spinellis is an associate professor in the Department of Management Science and Technology at the Athens University of Economics and Business, and the author of Code Reading; The Open Source Perspective (Addison-Wesley, 2003) and Code Quality: The Open Source Perspective (Addison-Wesley, 2006). His research interests include software engineering and programming languages. Spinellis received a PhD in computer science from Imperial College London. He is a member of the IEEE Computer Society and a senior member of the ACM. Contact him atdds@aueb.gr.

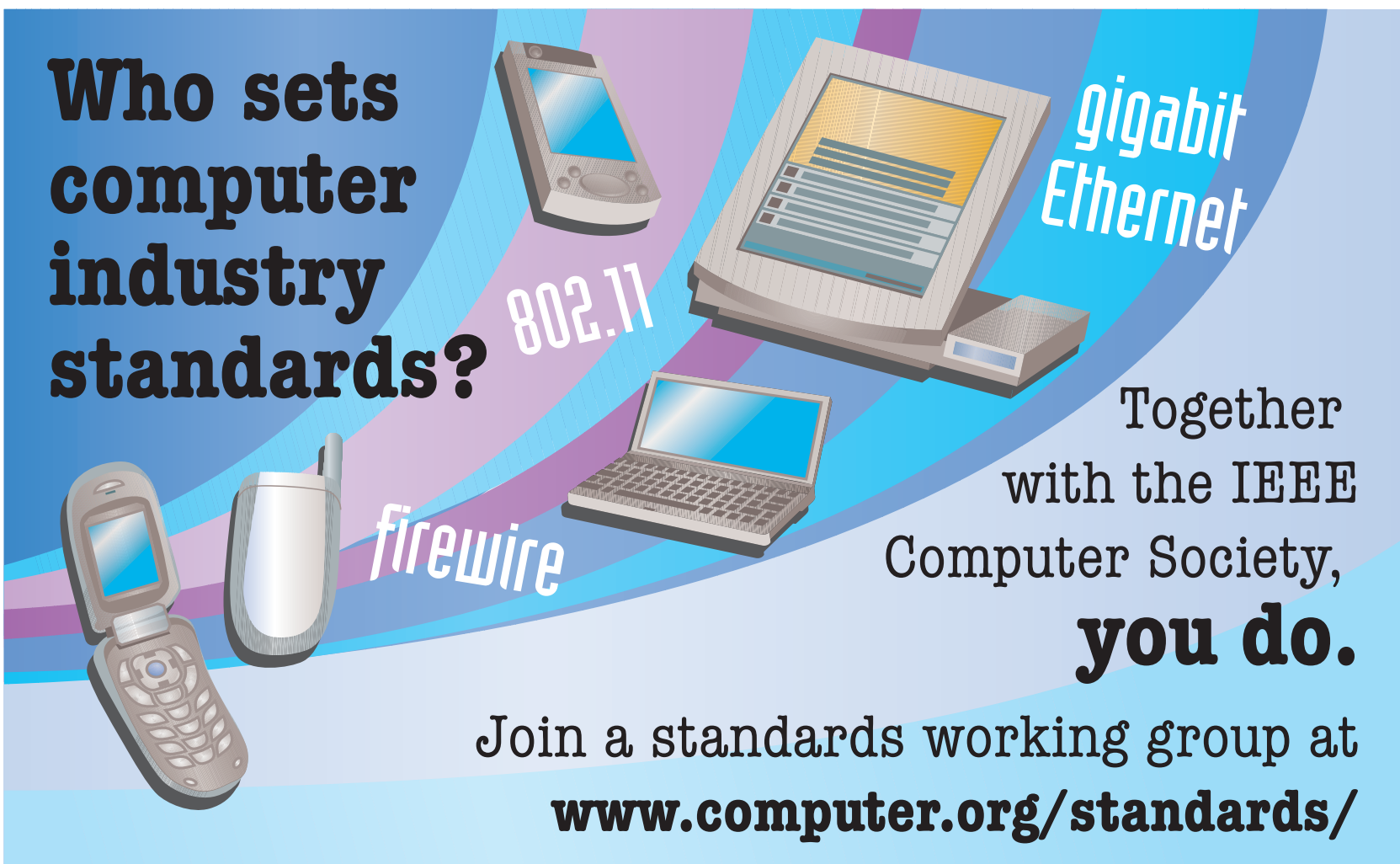

